\title{
Cardiopulmonary arrest and mortality trends, and their association with rapid response system expansion
}

\section{Jack Chen \\ MBBS, PhD, MBA(Exec) \\ Associate Professor. Simpson Centre for Health Services Research \\ Lixin Ou \\ MBA, MPH, PhD \\ Research Fellow, \\ Simpson Centre for Health \\ Service Research \\ Kenneth M Hillman \\ MD, FRCA, FCICM \\ Professor of Intensive Care \\ Arthas Flabouris \\ MD, FCICM, FANZCA \\ Intensivist, 2 and Clinical \\ Associate Professor ${ }^{3}$ \\ Rinaldo Bellomo \\ MD, FCICM, FRACP \\ Director of Intensive Care \\ Research ${ }^{4}$ \\ Stephanie J Hollis \\ BSc, MMedSc, PhD \\ Research Fellow, \\ Simpson Centre for Health \\ Services Research \\ Hassan Assareh \\ PhD, MEng, MSc(Manag) Research Fellow, \\ Simpson Centre for Health \\ Services Research \\ IUniversity of \\ New South Wales, \\ Sydney, NSW. \\ 2 Intensive Care, Royal Adelaide Hospital, \\ Adelaide, SA. \\ 3 School of Medicine University of Adelaide \\ Adelaide, SA. \\ 4 Austin Hospital \\ Melbourne, VIC}

jackchen@unsw.edu.au

MJA 2014; 201: 167-170 doi: 10.5694/mjal4.00019

Online first 28/07/14 erious adverse events such as cardiac arrests and preventable deaths are common in acute hospitals,,$^{1,2}$ with a conservative estimate of 200000 inhospital cardiopulmonary arrests (IHCAs) ${ }^{2}$ and up to 98000 potentially preventable deaths each year in the United States. ${ }^{1}$ Many IHCAs and unexpected deaths ${ }^{3}$ are preceded by physiological instability and deteriorating vital signs for some hours before the event. Accordingly, they are potentially preventable. A rapid response system (RRS) consists of an afferent arm to identify deteriorating hospital patients, and an efferent arm to respond to such deterioration by rapidly deploying a team led by a physician or a nurse with appropriate critical care skills. The RRS was introduced to prevent serious adverse events such as ICHA and unexpected death. 4

RRSs have now been implemented in many hospitals worldwide. ${ }^{5}$ There has been evidence for the effectiveness of RRSs from before-and-after studies, 6,7 multicentre studies 8,9 and systematic reviews. 5,10 However, further level 1 evidence will be difficult if not impossible to achieve, due to the challenging ethics of randomly allocating patients into those receiving early or delayed intervention, the ubiquitous nature of RRSs in many parts of the world, and methodological difficulties of randomising hospital clusters.

Population-based studies have been used to measure the associated change in patient outcomes across a region following system implementation, such as in trauma systems. ${ }^{11,12}$ A population-based study, therefore, may provide a real-world perspective on the change in major outcomes such as IHCA and hospital mortality during a period of RRS implementation among hospitals across a health service region. The aim of this study was to examine the change in IHCA incidence, IHCA-related mortality and hospital mortality rates among acute

\section{Abstrac}

Objectives: To understand the changes in the population incidence of inhospital cardiopulmonary arrest (IHCA) and mortality associated with the introduction of rapid response systems (RRSs).

Design, setting and participants: Population-based study of 9221138 hospital admissions in 82 public acute hospitals in New South Wales, using data linked to a death registry, from 1 January 2002 to 31 December 2009.

Main outcome measures: Changes in IHCA, IHCA-related mortality, hospital mortality and proportion of IHCA patients surviving to hospital discharge.

Results: RRS uptake increased from 32\% in 2002 to $74 \%$ in 2009 . This increase was associated with a 52\% decrease in IHCA rate, a 55\% decrease in IHCArelated mortality rate, a $23 \%$ decrease in hospital mortality rate and a $15 \%$ increase in survival to discharge after an IHCA (all $P<0.01$ ). The adjusted absolute reductions in IHCA-related mortality and hospital mortality were 1.49 $(95 \% \mathrm{Cl}, 1.30-1.68)$ and $4.05(95 \% \mathrm{Cl}, 3.17-4.76)$ patients per 1000 admissions, respectively. The decrease in IHCA incidence rate accounted for $95 \%$ of the reduction in IHCA-related mortality. In contrast, the increase in IHCA survival accounted for only $5 \%$ of the reduction in IHCA-related mortality.

Conclusions: During nearly a decade, as RRSs were progressively introduced, there was a coincidental reduction in IHCA, IHCA-related deaths and hospital mortality and an increased survival to hospital discharge after an IHCA. Reduced IHCA incidence, rather than improved postcardiac arrest survival, was the main contributor to the reduction in IHCA mortality.

hospitals within a large health jurisdiction in Australia, during a period of RRS expansion.

\section{Methods}

\section{Study design and sample}

Between 1 January 2002 and 31 December 2009, there were 84 acute public hospitals in New South Wales (total population, 7.3 million). We excluded two children's hospitals, patients younger than 14 years of age and studied the remaining 82 acute public hospitals (9221138 admissions). This study was approved by the New South Wales Population and Health Services Research Ethics Committee (LNR/11/CIPHS/64).

Patient outcomes and other related variables were derived from the NSW Admitted Patient Data Collection database, which includes the demographic and diagnostic information of each public and private hospital admission episode. All admissions to the study hospitals were linked to the NSW Registry of Births, Deaths and Marriages through the Centre for Health Record Linkage, which generated the unique de-identified person key for both datasets and forwarded the person key to the data custodians who provided the study investigators with the relevant data items. Two statewide telephone-based surveys (one in November 2007 and one in May 2013) and one web-based survey (October to December 2012) were conducted to ascertain the date of RRS implementation for every hospital. An RRS was defined as any system that had a clear objective in identifying and responding to the deteriorating hospital inpatients. A flowchart of the data-inclusion process is presented in Appendix 1; online at mja.com.au.

\section{Study outcomes}

The primary study outcomes were (i) IHCA incidence rate: the number of IHCAs divided by total number of admissions (including same-day admissions); (ii) IHCA-related mortality: the number of deaths among those patients who suffered an IHCA divided by the total number of admissions; and (iii) hospital mortality rate: the number of deaths divided by total number of hospital admissions. 
1 Trends for inhospital cardiopulmonary arrest (IHCA), 2002-2009

\begin{tabular}{|c|c|c|c|c|c|c|c|c|c|}
\hline & 2002 & 2003 & 2004 & 2005 & 2006 & 2007 & 2008 & 2009 & $P^{*}$ \\
\hline IHCA incidence rate ${ }^{\dagger}$ & 3.72 & 3.34 & 2.97 & 2.71 & 2.51 & 2.46 & 2.12 & 1.85 & $<0.001$ \\
\hline IHCA mortality rate ${ }^{\dagger}$ & 2.71 & 2.42 & 2.10 & 1.91 & 1.81 & 1.76 & 1.47 & 1.26 & $<0.001$ \\
\hline Hospital mortality rate ${ }^{\dagger}$ & 17.63 & 17.18 & 17.03 & 15.91 & 15.29 & 15.46 & 15.47 & 14.36 & $<0.001$ \\
\hline IHCA patients surviving to discharge & $27.2 \%$ & $27.6 \%$ & $29.1 \%$ & $29.6 \%$ & $27.9 \%$ & $28.4 \%$ & $30.8 \%$ & $32.3 \%$ & 0.002 \\
\hline 1-year postdischarge mortality after IHCA & $5.4 \%$ & $5.1 \%$ & $3.9 \%$ & $4.6 \%$ & $3.7 \%$ & $4.3 \%$ & $3.0 \%$ & $5.6 \%$ & 0.117 \\
\hline
\end{tabular}

The secondary study outcomes were (i) the proportion of IHCA patients surviving to discharge: the number of patients who survived to hospital discharge divided by total number of IHCA patients; and (ii) 1-year postdischarge mortality of IHCA patients: the number of deaths within 1-year after discharge alive from hospital among IHCA patients.

Death was defined as a patient documented as deceased within the Admitted Patient Data Collection database.

A cardiopulmonary arrest was identified from the International statistical classification of diseases and health related problems, 10th revision, Australian modification (ICD-10-AM) and defined as a state of pulselessness (I46) and/ or cessation of breathing (R09.2) that required cardiac massage, defibrillation or artificial ventilation.

A patient coded as I46 or R09.2 in any of the 54 non-principal diagnostic fields, but not coded for these as the principal diagnostic field, was defined as having had a cardiopulmonary arrest during hospitalisation. This process aimed to differentiate IHCA patients from patients admitted after an out-of-hospital cardiopulmonary arrest. NSW has implemented ICD10-AM since 1998. Each NSW acute public hospital has accredited coders who code data based on the patient charts. There were no changes for relevant diagnostic definitions and coding during the study period.

\section{Statistical analysis}

To evaluate changes in baseline characteristics by calendar year, we applied logistic regression, multinomial logistic regression and linear regression modelling for binary, categorical and continuous variables. To assess changes in each outcome over time, we assessed a linear trend for crude rates after excluding possible quadratic effect, using the study year as a continuous variable. We derived an adjusted trend for each outcome variable including calendar year as a categorical variable (with 2002 as the baseline reference year). We specified a Poisson distribution to directly estimate rate ratios instead of odds ratios in the models.13 A Huber-White sandwich estimator was used to account for hospital cluster effect. ${ }^{14}$ The adjusted rate ratio for each year (2003-2009) was multiplied by the observed rates for the reference year to obtain yearly risk-adjusted rates. These rates represent the estimated rates for each year if the patient case mix was identical to that in the reference year. In the adjusted model, we included year, age groups, sex, marital status, country of birth, socioeconomic status, geological area of hospitals (urban versus rural) and major hospital peer groups. We examined baseline risk groups with the Elixhauser method and patient comorbidities with the Charlson index based on ICD-10-AM coding. ${ }^{15}$ We examined the relationship between increased uptake of RRSs over the period studied and changes in rates of all study outcomes using Pearson correlation after assessing the linearity of all trends. We did not include baseline risk groups and the Charlson index in the adjusted model given recent reporting of potential biases introduced by these methods. ${ }^{16} \mathrm{~A} P$ value $<0.01$ was used to indicate statistical significance. All the analyses were conducted using Stata version 12 (StataCorp).

\section{Results}

Mean age increased and the proportion of female patients decreased over the study period (Appendix 2; online at mja.com.au). We observed a significant increase in the proportion of patients with renal failure, but a decrease in the proportion of patients with malignancy and chronic pulmonary diseases. No significant changes occurred in patients with cardiac arrhythmia, congestive heart failure, depression and metastatic solid tumours.

There was a consistent and significant secular trend in all hospitals toward a decrease in both crude and adjusted rates of IHCA, IHCA-related mortality and hospital mortality over the study period (Box 1, Box 2) (Appendix 3; online at mja.com.au). The adjusted rate reduction for IHCA was 1.93 (ie, $3.72-1.79$ ) patients per 1000 admissions (risk ratio [RR], 0.48; 95\% CI, 0.42-0.55; $P<0.001)$ between 2002 and 2009. The adjusted rate reduction in IHCA-related mortality was 1.49 (ie, $2.71-1.22$ ) patients per 1000 admissions (95\% CI, 1.30-1.68) (RR, $0.45 ; 95 \% \mathrm{CI}, 0.38-0.52 ; P<0.001)$. This accounted for $36.8 \%$ of the adjusted hospital mortality reduction: 4.05 patients (ie, 17.63 - 13.58) per 1000 admissions (95\% CI, 3.17-4.76) (RR, 0.77; 95\% CI: 0.73-0.82; P<0.001).

The adjusted proportion of IHCA patients surviving to hospital discharge increased in all hospitals from $27.2 \%$ to $31.3 \%$ over the study period $(4.1 \%$; RR, 1.15; 95\% CI, 1.02-1.29) (Box 3) (Appendix 3; online at mja.com.au). In 2009 , the overall reduction in mortality due to decreased mortality after IHCA was $0.073(4.1 \% \times 1.79)$ deaths per 1000 admissions. Such reduction accounted for only $1.8 \%(0.073 / 4.05)$ of total hospital mortality improvement and only $4.9 \%(0.073 / 1.49)$ of total improvement in IHCA-related mortality. The proportion of IHCA patients who were still alive 1 year after suffering an IHCA fluctuated around 5\%.

Before 1 January 2002, 19 (23.2\%) of 82 hospitals had an RRS. The proportion of the study hospitals having an RRS progressively increased from $26(31.7 \%)$ in 2002 to 61 (74.4\%) in 2009. There was a close association between the proportion of hospitals 
2 Risk-adjusted incidence rates of inhospital cardiopulmonary arrest (IHCA), IHCA-related mortality and hospital mortality, and proportion of hospitals with a rapid response system (RRS), 2002-2009

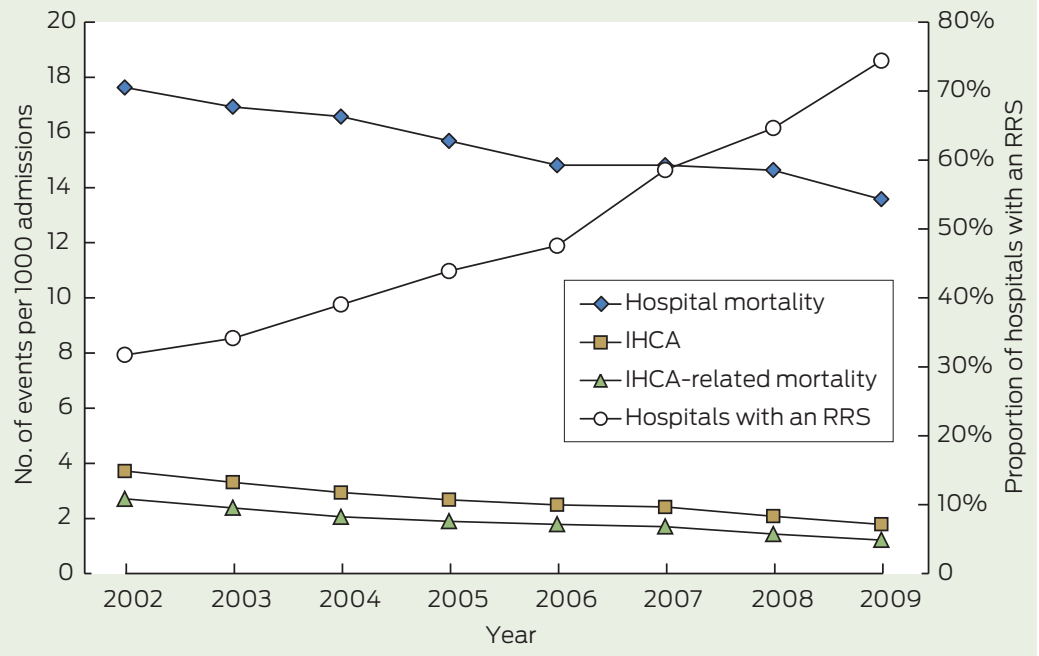

3 Risk-adjusted proportion of inhospital cardiopulmonary arrest (IHCA) patients surviving to hospital discharge and 1-year postdischarge mortality after IHCA, 2002-2009

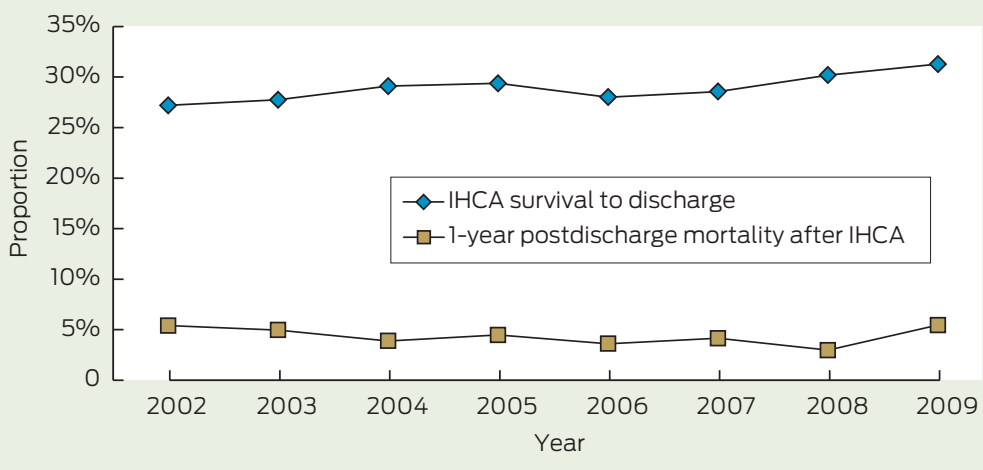

with an RRS and the adjusted IHCA rate $(-0.95 ; P<0.001)$; the adjusted IHCA-related mortality rate $(-0.95$; $P<0.001) ;$ and the adjusted hospital mortality rate $(-0.94 ; P<0.001)$. There was a positive association between implementation of an RRS and the proportion of IHCA patients surviving to discharge $(0.84 ; P=0.009)$ but no association with 1-year postdischarge mortality among IHCA patients $(-0.19$; $P=0.65)($ Box 2, Box 3).

\section{Discussion}

We found that the proportion of hospitals with an RRS more than doubled over the 8-year study period across 82 acute NSW hospitals. Over the same period, there was also $>50 \%$ reduction in the IHCA incidence rate; $>50 \%$ reduction in IHCA-related mortality;
$23 \%$ overall reduction in hospital mortality; and $4 \%$ increase in the proportion of IHCA patients who survived to discharge. However, there was no change in 1-year postdischarge mortality among IHCA patients. Moreover, the reduction in IHCA incidence rate and the increase in IHCA survival accounted for $95 \%$ and $5 \%$ of the IHCA-related mortality reduction, respectively.

The significant improvement in IHCA rate shown in the current study while RRS services were being expanded is broadly consistent with that reported in a recent meta-analysis supporting the external validity of our findings. ${ }^{5}$ Our study also demonstrated a reduction in adult IHCA-related mortality of 1.49 per 1000 admissions. This finding is consistent with reports from two cluster randomised controlled trials8,17 and most observational before-and-after studies, ${ }^{9}$ but is in contrast with the findings of a recent meta-analysis. ${ }^{10}$ This discrepancy may be due to methodological limitations within the meta-analysis, such as mistaking data on "unexpected deaths" as overall mortality when including outcomes from the largest previous RRS study. ${ }^{18}$ Our finding that the proportion of IHCA patients surviving to discharge improved over the study period is also consistent with previous findings. ${ }^{19}$

Despite initial enthusiasm and resources devoted to patient safety, the influence of patient safety initiatives has been slow and disappointing, 20 with little demonstrable impact on patient outcomes such as mortality. ${ }^{21} \mathrm{An}$ RRS is a different approach to patient safety in acute hospitals. The implementation process involves changes across the health service system aimed at improving patient safety. The compelling intuitive appeal and local acceptance of RRSs have driven their expansion in hospitals around the world.

Based on our findings, and assuming RRS implementation trends and changes in IHCA and hospital mortality rates similar to those of our study, the estimated number of lives saved due to the reduction in annual IHCArelated mortality would be over 12800 in Australia in 2011 compared with 2002.22 To provide a context for such notional improvement, the overall yearly mortality of other conditions for which preventive strategies are applied such as lung, bowel, prostate and breast cancer is well below such numbers. Similarly, assuming an IHCA incidence of 200000 per year, with a $20 \%$ survival rate, 80000 cardiac arrest-associated deaths might potentially be prevented each year in the US.

Previous research around cardiac arrests has largely been devoted to identifying and implementing more effective ways of delivering cardiopulmonary resuscitation (CPR). ${ }^{2}$ Despite many years of research and the enormous cost of delivering CPR services, ${ }^{23}$ there has been slow and limited improvement in outcome. ${ }^{24}$ In contrast, we have shown that the prevention of IHCA resulted in $95 \%$ of the reduction in IHCA-related mortality, suggesting the importance of concentrating 
our efforts and funding on systems to prevent arrests.

Rapid response teams issue more not-for-resuscitation (NFR) orders than conventional cardiac arrest teams. ${ }^{25}$ Thus, implementing an RRS may artificially improve IHCA-related outcomes by excluding patients with an NFR order. If this were the case, a decrease in IHCA should be associated with unchanged or increased overall hospital mortality. In contrast, our study found a simultaneous improvement in all outcome indicators, including hospital mortality. This finding challenges speculations that implementing an RRS may introduce unintended and adverse systematic distortions in patient management. The stable trend of 1-year postdischarge mortality suggests that there was no increased proportion of IHCA patients with an NFR order who were discharged to die in the community over the study period. As expected, the unchanged 1-year mortality also implies that RRSs do not improve the long-term health outcomes of patients who survive an IHCA.

This is the first population-based study of the implementation of an RRS spanning almost a decade. It used a well established definition of IHCA based on an existing large dataset. The outcomes measured, such as IHCAs and hospital deaths, were robust and standardised across the jurisdiction, generalisable and ascertained by independent professional coders. For the first time in RRS-related studies, the 1-year postdischarge mortality after IHCA was estimated through a population-based data linkage. Further, the study showed the feasibility of assessing complex system interventions at the population level and provides a set of outcome indicators that can be used to track hospital performance and safety.

Our study has limitations. First, it is an observational study and, as such, we cannot assume any causality for the relationships identified. In addition, we cannot exclude a beneficial effect on outcomes from other patient safety initiatives or secular trends in hospital performance. Also, we cannot rule out the possibility that the increased use of advanced cardiological medical and interventional treatments during the past decade might have contributed to the reduction of both IHCA incidence and related mortality. Our results would benefit from confirmation in other studies. However, there were no other state-wide initiatives specifically targeted to the prevention of IHCA during the study period. To our knowledge, there were no other major changes in the way health care was delivered in hospitals over the study period. For example, the state's hospitals remain staffed by specialist physicians and patient care has not moved to a hospitalist or acute general physician model. There was little change in community management of patients who may be at the end of life, to perhaps change the nature of the hospital population. Nor was there any major uptake of electronic patient records during that period. Finally, we did not explore the individual impact of the various hospital RRS implementation strategies or of RRS team composition and structure.

In summary, our study provides a population-based estimate, in a large health care jurisdiction, of the IHCA and hospital mortality trends during a period of RRS implementation. We found a significant association between the $50 \%$ reduction in both IHCA and IHCA-related mortality and the more than doubling in RRS uptake. Moreover, we found that the decrease in IHCA-related mortality was due to prevention and not to advances in cardiopulmonary arrest resuscitation. This study highlights the utility of population-based studies in examining secular changes in patient outcomes, their association with changes in patient safety systems over time, and the importance of reporting performance measures related to prevention of IHCAs when evaluating interventions targeted at reducing IHCA-related mortality.

Acknowledgements: Our study was supported by National Health and Medical Research Council project grants APP1009916 and APP1020660.

\section{Competing interests: No relevant disclosures.}

Received 6 Jan 2014, accepted 22 May 2014.

1 Institute of Medicine. To err is human: building a safer health system. Washington, DC: National Academies Press, 2000.

2 Morrison LJ, Neumar RW, Zimmerman JL, et al. Strategies for improving survival after in-hospital cardiac arrest in the United States: 2013 consensus recommendations: a consensus statement from the American Heart Association. Circulation 2013; 127: 1538-1563.

3 Kause J, Smith G, Prytherch D, et al. A comparison of antecedents to cardiac arrests, deaths and emergency intensive care admissions in Australia and New Zealand, and the United Kingdom -the ACADEMIA study. Resuscitation 2004; 62: 275-282.

4 Devita MA, Bellomo R, Hillman K, et al. Findings of the first consensus conference on medical emergency teams. Crit Care Med 2006; 34: 2463-2478.

5 Winters BD, Weaver SJ, Pfoh ER, et al. Rapid-response systems as a patient safety strategy: a systematic review. Ann Intern Med 2013; 158 (5 Pt 2): 417-425.

6 Bellomo R, Goldsmith D, Uchino S, et al. A prospective before-and-after trial of a medical emergency team. Med J Aust 2003; 179: 283-287.

7 Buist MD, Moore GE, Bernard SA, et al. Effects of a medical emergency team on reduction of incidence of and mortality from unexpected cardiac arrests in hospital: preliminary study. BMJ 2002; 324: 387-390.

8 Chen J, Bellomo R, Flabouris A, et al. The relationship between early emergency team calls and serious adverse events. Crit Care Med 2009; 37: 148-153.

9 Jones DA, Bagshaw SM, Barrett J, et al. The role of the medical emergency team in end-of-life care: a multicenter, prospective, observational study. Crit Care Med 2012; 40: 98-103.

10 Chan PS, Jain R, Nallmothu BK, et al. Rapid response teams: a systematic review and meta-analysis. Arch Intern Med 2010; 170: 18-26.

11 Mullins RJ, Mann NC. Population-based research assessing the effectiveness of trauma systems. J Trauma 1999; 47 (3 Suppl): S59-S66.

12 Cameron PA, Gabbe BJ, Cooper DJ, et al. A statewide system of trauma care in Victoria: effect on patient survival. Med J Aust 2008; 189: 546-550.

13 Greenland S. Model-based estimation of relative risks and other epidemiologic measures in studies of common outcomes and in case-control studies. Am J Epidemiol 2004; 160: 301-305.

14 White H. Maximum likelihood estimation of misspecified models. Econometrica 1982; 50: 1-26. doi: 10.2307/1912004

15 Quan H, Sundararajan V, Halfon P, et al. Coding algorithms for defining comorbidities in ICD-9-CM and ICD-10 administrative data. Med Care 2005; 43 . 1130-1139.

16 Wennberg JE, Staiger DO, Sharp SM, et al. Observational intensity bias associated with illness adjustment: cross sectional analysis of insurance claims. BMJ 2013; 346: f549.

17 Priestley G, Watson W, Rashidian A, et al. Introducing Critical Care Outreach: a ward-randomised trial of phased introduction in a general hospital. Intensive Care Med 2004; 30: 1398-1404.

18 Hillman K, Chen J, Cretikos M, et al. Introduction of the medical emergency team (MET) system: a cluster-randomised controlled trial. Lancet 2005; 365: 2091-2097.

19 Girotra S, Nallamothu BK, Spertus JA, et al. Trends in survival after in-hospital cardiac arrest. NEngl J Med 2012; 367: 1912-1920.

20 Leape LL, Berwick DM. Five years after To Err Is Human: what have we learned? JAMA 2005; 293: 2384-2390.

21 Benning A, Dixon-Woods M, Nwulu U, et al. Multiple component patient safety intervention in English hospitals: controlled evaluation of second phase. BMJ 2011; 342: d199.

22 Australian Institute of Health and Wellfare. Australian hospital statistics 2011-12. Canberra: AlHW, 2013. (AlHW Cat. No. HSE 134; Health Services Series 50.) http://www.aihw.gov.au/publicationdetail/?id=60129543133 (accessed May 2014).

23 Lee KH, Angus DC, Abramson NS. Cardiopulmonary resuscitation: what cost to cheat death? Crit Care Med 1996; 24: 2046-2052.

24 Nolan JP. Optimizing outcome after cardiac arrest. Curr Opin Crit Care 2011; 17: 520-526.

25 Chen J, Flabouris A, Bellomo R, et al. The Medical Emergency Team System and not-for-resuscitation orders: results from the MERIT study. Resuscitation 2008; 79: 391-397. 